Revue des patrimoines

\title{
Un monument sur la frontière : commémorer la guerre de 1870 à Mars-la-Tour (1871-1914)
}

A monument on the border, commemorating the Franco-Prussian war at Marsla-Tour (1871-1914)

\section{Benoît Vaillot}

\section{OpenEdition}

\section{Journals}

Édition électronique

URL : http://journals.openedition.org/insitu/20090

DOI : 10.4000/insitu.20090

ISSN : 1630-7305

Éditeur

Ministère de la culture

Référence électronique

Benoît Vaillot, « Un monument sur la frontière : commémorer la guerre de 1870 à Mars-la-Tour

(1871-1914) », In Situ [En ligne], 38 | 2019, mis en ligne le 15 février 2019, consulté le 19 avril 2019

URL : http://journals.openedition.org/insitu/20090 ; DOI : 10.4000/insitu.20090

Ce document a été généré automatiquement le 19 avril 2019

\section{c)}

In Situ Revues des patrimoines est mis à disposition selon les termes de la licence Creative Commons Attribution - Pas d'Utilisation Commerciale - Pas de Modification 4.0 International. 


\title{
Un monument sur la frontière : commémorer la guerre de 1870 à Mars-la-Tour (1871-1914)
}

\author{
A monument on the border, commemorating the Franco-Prussian war at Mars- \\ la-Tour (1871-1914)
}

Benoît Vaillot

1 «Ô France! N'oublie jamais ceux qui sont morts pour toi. Reviens toujours dans ces lieux dont les noms autrefois obscurs sont désormais immortels ${ }^{1}$. »

2 Entre les 14 et 18 août 1870 , le pays messin est ravagé par plusieurs batailles, qui laissent plus de 75000 morts parmi les combattants français et allemands ${ }^{2}$. Après la guerre, la mémoire française a conservé les noms de Borny-Colombey, Mars-la-Tour et Saint-Privat ${ }^{3}$ - Ces lieux dessinent à partir de 1871 une géographie mémorielle transfrontalière. En effet, le traité de Francfort fait passer la nouvelle frontière franco-allemande au milieu des champs de bataille lorrains. Lors des négociations, Otto von Bismarck demande leur annexion en raison des pertes considérables qu'y subit l'armée prussienne ; Guillaume $\mathrm{I}^{\mathrm{er}}$ de Prusse parlait d'ailleurs à ce sujet de «tombeau de sa garde». Les lieux des affrontements rejoignent l'Alsace-Lorraine, à l'exception de Mars-la-Tour qui reste français.

3 Ce petit village lorrain, où se déroulèrent les combats les plus meurtriers, le 16 août 1870, devient un véritable «lieu de mémoire» où se construit l'identité des survivants ${ }^{4}$. Son monument commémoratif de la guerre (fig. 1), à moins d'un kilomètre de la frontière allemande, constitue un cas original de patrimonialisation. Il témoigne du passé et se veut être un rappel aux générations futures des martyrs tombés pour la patrie. Le culte des morts réitéré tous les ans au même moment et au même endroit, avec des participants venant de toute la Lorraine, et même au-delà, participe pleinement à la création d'une communauté imaginée ${ }^{5}$. 
Figure 1

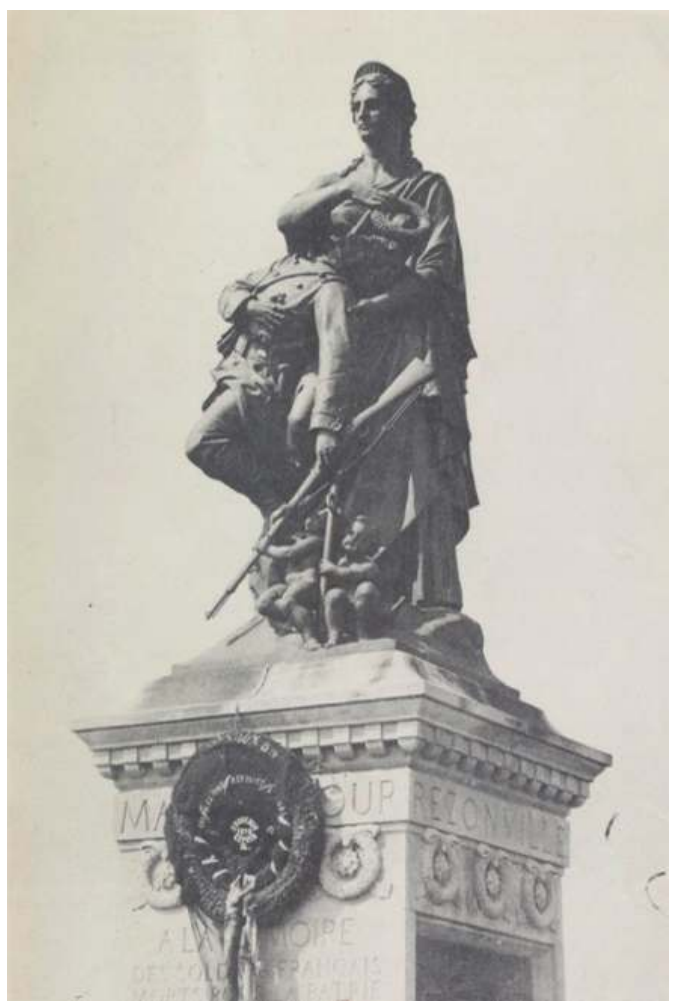

Photographie du monument, J. Royer, Nancy, reproduite dans LEROY, Osvald. Mars-la-Tour, 16-18 août 1870. Paris : Fischbacher, 1887

4 Les politiques du souvenir mises en œuvre au XIX siècle ont tendance à être minorées par l'historiographie des commémorations de la guerre, à l'exception des travaux de Stéphane Tison ${ }^{6}$. En effet, on considère que jusqu'à la Première Guerre mondiale, les monuments commémoratifs n'ont de résonance que locale ${ }^{7}$. Mieux encore, Antoine Prost avance que les monuments aux morts de la guerre de 1870 ne sont pas édifiés dans l'émotion du deuil national, mais vingt ou trente ans plus tard, après l'épisode boulangiste et au début du $\mathrm{xx}^{\mathrm{e}}$ siècle ${ }^{8}$. Il souligne le rôle du Souvenir Français, association spécialement fondée pour entretenir la mémoire des soldats morts, et affirme que ces monuments émanent «de la partie de l'opinion publique qui cultivait la volonté de revanche. Ils n'engagent ni l'ensemble de la nation, ni ses représentants officiels, collectivités locales ou État. $»^{9}$. Rémi Dalisson va plus loin : il assimile toute forme de commémoration de la guerre de 1870 à du revanchisme et fait de l'État son principal $\operatorname{artisan}^{10}$.

5 La présente étude a plusieurs objectifs : contribuer à l'histoire de la patrimonialisation des frontières, revisiter l'historiographie des commémorations de guerre en France et enfin, rendre compte d'un monument en interrogeant les pratiques, les discours, et les significations qui l'ont accompagné. À la suite des travaux de Karine Varley sur la mémoire de la guerre franco-allemande en France ${ }^{11}$, nous proposons une histoire par en bas des pratiques commémoratives de la guerre à travers Mars-la-Tour et son monument 12 .

6 Nous souhaitons restituer la genèse d'un monument national dont l'initiative revient à la population locale, et qui a été progressivement récupéré par l'État. L'attention portée à 
l'emboitement des significations du monument de Mars-la-Tour lors de son inauguration permet d'illustrer les tensions qu'il a pu y avoir entre le centre et la périphérie. Afin de comprendre comment on s'approprie et comment vit un patrimoine mémoriel à la frontière, les pratiques commémoratives font l'objet d'une analyse à part entière. Enfin, nous interrogeons le sens que peut revêtir un tel monument à travers ses usages.

\section{Un monument national d'initiative locale investi par l'État}

7 Dans l'immédiat après-guerre, non loin des champs de bataille, se manifeste à la frontière un culte des morts orchestré par le clergé local à destination des habitants et des proches des disparus, où se mêlent étroitement sentiment national et sentiment religieux.

Le 16 août 1872, l'abbé Stef, curé de Mars-la-Tour, célèbre devant ses huit cents ouailles une messe solennelle en l'honneur des soldats morts pour la patrie ${ }^{13}$. Une procession se forme jusqu'au cimetière où sont inhumés les soldats français tombés non loin, deux ans auparavant ${ }^{14}$. Alors que les troupes allemandes occupent encore la Meurthe-et-Moselle, on se souvient, dans l'intimité du cimetière municipal, de la guerre qui s'est déroulée au village. La cérémonie est reconduite en 1873 et, avec le reliquat des offrandes du service funèbre, naît l'idée d'élever une croix ${ }^{15}$. Une première souscription est lancée, et son succès est tel qu'est formé un comité local pour ériger un monument. À sa tête, on retrouve le maire, le curé ainsi que toute la municipalité1 ${ }^{16}$. L'idée première est de construire une pyramide ou une colonne ${ }^{17}$. Cette manifestation spontanée de patriotisme se fait de façon tout à fait indépendante des autorités ${ }^{18}$.

Cependant, l'État va devoir tenir compte de cette initiative locale afin de tenir ses engagements internationaux. En effet, l'article 16 du traité de Francfort enjoint les gouvernements français et allemand à faire respecter et entretenir les tombeaux des soldats ensevelis sur leurs territoires respectifs. Il est transposé dans le droit français par la loi du 4 avril 1873. Comportant une crypte militaire, le monument de Mars-la-Tour est ainsi un moyen d'appliquer la loi. Le sculpteur Frédéric-Louis-Désiré Bogino (1831-1899), spécialisé dans la statuaire religieuse, propose un projet, trop coûteux à mettre en œuvre 19. Un comité du monument, piloté par le ministère de la Guerre, se constitue alors à Paris pour soutenir l'initiative locale, rassembler des fonds et réceptionner l'œuvre ${ }^{20}$. Le général Paul de Ladmirault, vétéran de la bataille de Mars-la-Tour, livre à Bogino quatre pièces de canon hors service ${ }^{21}$. De cette manière, l'État allège considérablement le coût du monument et traite directement avec le sculpteur. Les Malatouriens se retrouvent à la fois soutenus et dépossédés de leur initiative.

10 Le monument de Mars-la-Tour est construit en 1875, près de la frontière, sur un terrain offert à la municipalité par un particulier ${ }^{22}$. Sa propriété est l'objet d'un long contentieux entre l'État et le comité local, et indique déjà qu'un monument national n'est pas nécessairement étatique ${ }^{23}$. À mi-chemin entre une descente de croix et le Gloria Victis d'Antonin Merciée ${ }^{24}$, la statue de Bogino laisse à voir une France debout et résignée (voir fig. 1). Elle tient dans ses bras un soldat mortellement blessé dont le fusil, qui lui échappe de la main, est saisi par un enfant. À ses côtés se tient un autre enfant, debout sur une ancre, symbole d'espoir. Sur le monument on peut lire, entouré des noms de Rezonville, Gravelotte, Saint-Privat et Mars-la-Tour : 


\section{À LA MÉMOIRE \\ DES SOLDATS FRANÇAIS \\ MORTS POUR LA PATRIE \\ DANS LES JOURNÉES \\ DES 16 ET 18 AOÛT 1870}

Le monument est flanqué de deux hauts-reliefs, «Le combat d'infanterie » (fig. 2) et « Le combat de cavalerie » (fig. 3), qui représentent les chefs militaires - dont plusieurs membres du comité national - dans des postures édifiantes de commandement ou de mort héroïque. Ce sont des illustrations d'un récit de René de La Tour du Pin, membre de l'état-major illustrant une posture héroïque : «C'était horrible et merveilleux! ${ }^{25}$. Le comité local n'est pas à l'origine de ces deux pièces, ajoutées deux ans après la construction $^{26}$. Le gouvernement glorifie l'armée et ses chefs à l'endroit même où les populations à la frontière peuvent témoigner de la défaite. Ce que les hauts-reliefs donnent à voir, c'est bien la guerre vue par l'état-major français.

Figure 2

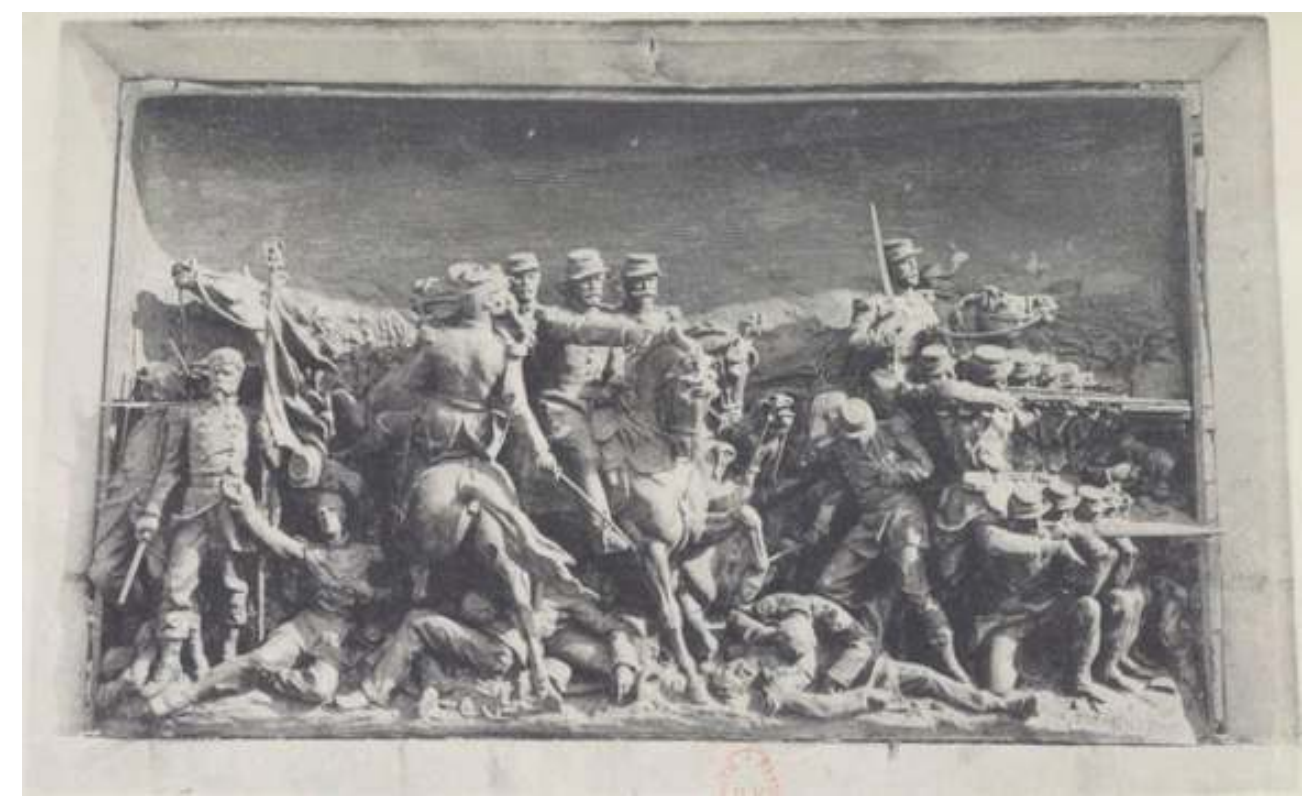

Photographie du haut-relief « Le combat d'infanterie », J. Royer, Nancy, reproduite dans LEROY, Osvald. Mars-la-Tour, 16-18 août 1870. Paris : Fischbacher, 1887. 


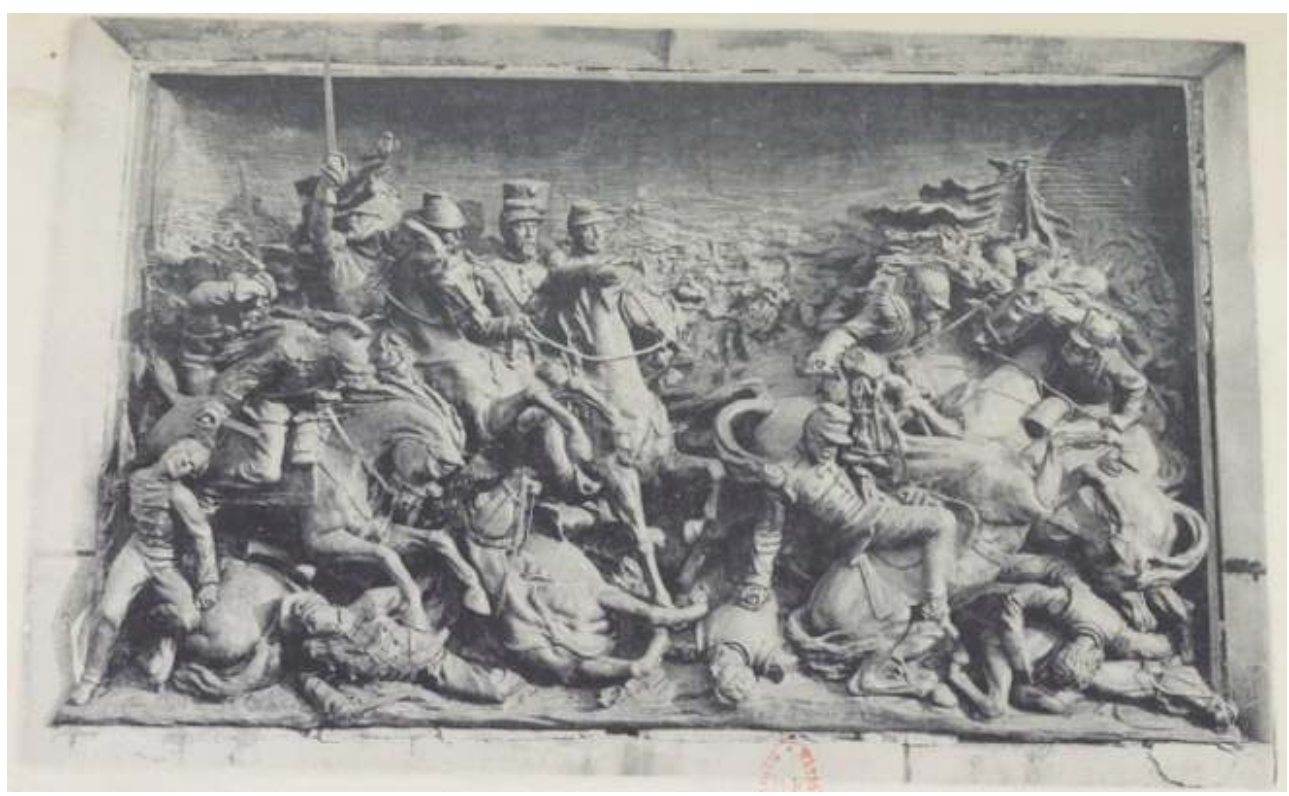

Photographie du haut-relief « Le combat de cavalerie », J. Royer, Nancy, reproduite dans LEROY, Osvald. Mars-la-Tour, 16-18 août 1870. Paris : Fischbacher, 1887.

12 Si l'on veut avoir une idée de la façon dont la mémoire locale s'est saisie des événements de la guerre, il faut s'intéresser à la transformation de l'église paroissiale en église commémorative. En 1877, le successeur de l'abbé Stef, l'abbé Joseph Faller (1834-1914), un optant $^{27}$ né à Metz, redécore l'intérieur de l'église, puis fait rehausser le clocher de huit mètres et le surmonte d'une plate-forme carrée pour que l'on puisse bénéficier d'un panorama sur les anciens champs de bataille ${ }^{28}$. Le décor polychrome du maitre-autel, par exemple, représente un aumônier militaire portant le brassard des ambulanciers; il assiste aux derniers moments d'un jeune sergent du $1^{\text {er }}$ régiment d'infanterie ${ }^{29}$. Cette scène illustre le souvenir des populations, non pas la bataille, mais l'après-bataille : les ambulances, les blessés que l'on soigne et les morts que l'on enterre. C'est le moment où l'on inhume au cimetière municipal, auprès des aïeux de la communauté villageoise, ces inconnus qui font partie d'une communauté plus grande, de la communauté imaginée ${ }^{30}$. Les murs affichent ça et là des petites plaques commémorant les soldats individuellement ou collectivement, sans hiérarchie de grades, parfois agrémentées d'un ultima verba patriotique et chrétien.

Sur ordres du ministre de l'Intérieur, les ossements sont exhumés du cimetière en 1875 et réunis dans la crypte située sous le monument, à trois mètres cinquante sous terre ${ }^{31}$. L'entrée est fermée par une grille, cachée par le gazon ${ }^{32}$. L'initiative locale visant à construire un monument aux morts pour la patrie a été récupérée par l'État qui dispose d'un agenda commémoratif propre, différent de celui des populations vivant à la frontière. Cette discordance est pleinement mise en lumière lors de l'inauguration du monument. 


\section{Une inauguration sous contrôle ou les débuts difficiles d'un dialogue entre le centre et la périphérie}

14 La double inauguration du monument de Mars-la-Tour permet de mettre en lumière un dialogue entre l'État et les populations frontalières au sujet de la commémoration des morts de la guerre de 1870. Un dialogue qui aurait pu ne pas avoir lieu, car il est même envisagé de renoncer au monument pour éviter une crise diplomatique avec l'Allemagne ${ }^{33}$ . Le monument national est finalement inauguré en deux temps : une première fois en 1875 , lors de son achèvement, et une seconde fois en 1877 , lors de la pose des hauts-reliefs évoqués plus haut.

Le gouvernement d'Ordre moral s'oppose fermement à ce que l'inauguration ait lieu le 16 août 1875 , contre le désir du comité local qui souhaitait continuer à célébrer l'anniversaire de la bataille ${ }^{34}$. Les Malatouriens se voient dépouillés de leur initiative en raison du contexte diplomatique de "Krieg in Sicht » : le gouvernement allemand menace d'attaquer préventivement la France dont le relèvement rapide inquiète ${ }^{35}$. Le ministre de l'Intérieur demande l'ajournement de l'inauguration ${ }^{36}$ au motif que l'« Allemagne a appris avec un vif déplaisir l'érection du monument de Mars-la-Tour $"^{37}$. On craint à Paris que les participants à la commémoration ne témoignent de leur patriotisme et poussent des cris hostiles, «À bas l'Allemagne, à bas Bismarck ! » ou même seulement «Vive la France ! " Une peur particulièrement motivée par les populations des territoires annexés qui, comme les années précédentes, passent la frontière pour se rendre à Mars-la-Tour ${ }^{39}$. Il est jugé plus sage d'inaugurer le monument le 2 novembre 1875, jour des Défunts, où l'on bénit les tombes ${ }^{40}$. Toute pompe est soigneusement évitée: aucun discours officiel, interdiction de revêtir l'uniforme militaire ${ }^{41}$, pas de musique militaire ${ }^{42}$, et le préfet envisage de s'abstenir d'y participer ${ }^{43}$ L'événement est réduit à sa stricte dimension religieuse. Le sous-préfet de Briey fait même démolir la charpente de l'estrade érigée

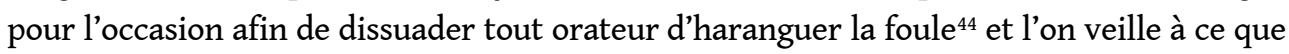
le pavoisement soit réduit au strict minimum ${ }^{45}$.

Le 26 septembre 1877, le comité local inaugure une chapelle funéraire et un arc commémoratif dans l'église paroissiale, réalisés grâce aux souscriptions des parents et amis des soldats disparus ${ }^{46}$. Le comité de Paris choisit la même date pour inaugurer les hauts-reliefs du monument. Un grand nombre de généraux et d'officiers se déplace pour l'événement ${ }^{47}$. Alors que le maire de Mars-la-Tour demande un détachement d'hommes en armes pour assister à la bénédiction de la chapelle ${ }^{48}$, le ministre de l'Intérieur demande à nouveau que la cérémonie soit uniquement religieuse ${ }^{49}$, et que le préfet se garde de prendre la parole ${ }^{50}$. Un important dispositif de police, visant à maintenir « le bon ordre ", est déployé par crainte de débordements ${ }^{51}$.

Quelques discours, très courts, témoignent de la politique conservatrice du gouvernement en 1875 et 1877. Qu'ils soient prononcés par le clergé local ou les représentants de l'État, ils visent à rétablir l'ordre moral. Les éventuelles aspirations à la Revanche sont balayées par la proclamation de l'honneur de l'Armée et les nécessités d'entretenir des relations apaisées avec l'ennemi d'hier ${ }^{52}$. Les soldats sont morts en martyrs pour la patrie et sont les rédempteurs d'une France qui renoue avec les «vraies valeurs»: une sorte d'holocauste nécessaire ${ }^{53}$. Les seuls discours qui s'écartent de cette ligne sont ceux des élus locaux, vétérans de la guerre de $1870^{54}$. L'inauguration se termine par quelques «Vive la France! », malgré l'action de l'État qui cherche à les contenir. 

allemande se réunissent au pied du monument national et entretiennent la flamme du patriotisme lorrain. Tous les ans, par voie de presse, les Lorrains sont invités à célébrer l'anniversaire des 16 et 18 août $1870^{60}$. On ne manque jamais d'y joindre les horaires de trains en provenance de Nancy et de Metz; les autorités allemandes se plaignent régulièrement qu'à la mi-août, les trains en direction de la frontière soient bondés tandis que la Compagnie des chemins de fer de l'Est affrète des trains supplémentaires ${ }^{61}$.

Au lendemain de l'Assomption, on se rend en pèlerinage à Mars-la-Tour, vêtu de noir en signe de deuil. Les pèlerins viennent de toute la région, et en particulier de Metz et ses environs - c'est-à-dire de territoires allemands -, munis de bouquets de pensées et de couronnes d'immortelles ${ }^{62}$. Des alentours immédiats, on vient à pied ou en train toujours bondé, tandis que les très nombreuses voitures et bicyclettes ${ }^{63}$ stationnent dans la rue principale du village, pavoisée pour l'occasion ${ }^{64}$. Ceux qui viennent de loin séjournent dans les rares auberges ou chez l'habitant, et les pouvoirs publics réquisitionnent parfois des bâtiments pour les vétérans ${ }^{65}$. Au passage de la frontière et devant les douaniers et 
militaires allemands qui patrouillent, les hommes mettent à leur veste des cocardes, et les femmes des rubans tricolores à leur chevelure ${ }^{66}$.

Selon un rite immuable, l'abbé Faller dirige à midi un service religieux sobre en «mémoire des soldats français morts pour la patrie dans les journées des 16 et 18 août $1870 \aleph^{67}$. Plusieurs orateurs viennent en chaire prononcer un sermon patriotique. Et c'est en direction du maître-autel, où il est inscrit «Honneur et Patrie ", à l'est, sous les drapeaux tricolores reliés par un large crêpe noir, que l'on communie. Ce temple patriotique ne suffit pas toujours à accueillir la nombreuse assistance. On déplore même une bousculade, en 1889 , que le piquet de gendarmes et douaniers n'a pu contenir ${ }^{68}$. Une quête pour l'entretien de tout ce patrimoine mémoriel est assurée par deux jeunes filles, en costume lorrain et alsacien ${ }^{69}$.

La municipalité, le maire et le curé se mettent alors en marche et conduisent une procession de plusieurs milliers de personnes jusqu'au monument pavoisé pour l'occasion. Le clergé y bénit le lieu sacré en en faisant deux fois le tour. Des personnalités lorraines d'envergure nationale sont invitées à la cérémonie et prononcent des discours ponctués de «Vive la France! » et de «Vive l'Armée !». La musique d'un régiment local est détachée par le gouvernement, beaucoup plus sensible aux demandes locales à mesure que le régime se républicanise. La Marseillaise retentit alors sous les clameurs patriotiques ; on joue aussi des airs patriotiques comme Alsace et Lorraine $e^{70}$, chants encore considérés il y a peu comme appartenant au répertoire des cafés-concerts parisiens et sans écho populaire ${ }^{71}$.

Les élus locaux, les sociétés - celles de vétérans avant tout - se disputent l'honneur de déposer des couronnes mortuaires au pied du monument, dans l'espace circonscrit par des grilles qui délimitent la superficie à fleurir ${ }^{72}$. Chacun peut laisser son petit bouquet au-dessus de la crypte où repose peut-être un fils, un parent, un proche. On peut y voir des rubans où il est écrit «Metz! Espoir !» ou encore «A. L.», pour Alsace-Lorraine ${ }^{73}$. Enfin, le clergé exhorte la foule à entonner les prières liturgiques de l'absoute ${ }^{74}$. Les invités officiels, dont l'importance sur le plan national va croissant au fil du temps, se voient ensuite offrir une collation à la mairie, tandis que, sur la plaine, on dresse des tables, on banquette et on pique-nique en profitant de l'été ${ }^{75}$. Les enfants peuvent alors s'amuser et faire exploser quelques pétards, qui ne manquent pas d'attiser la fébrilité des douaniers et des militaires qui sillonnent la frontière.

L'infatigable abbé Faller collectionne les vestiges de la guerre qu'il fait exposer dans l'église paroissiale jusqu'à ce qu'il les rassemble dans un musée, en 1902, qu'il cède à la municipalité en $1907^{76}$. Le pèlerin peut associer le souvenir abstrait de la guerre à une réalité matérielle : on lui donne à voir des armes, mais aussi des gamelles et des gourdes perforées $^{77}$. La muséographie laisse une grande place aux histoires individuelles: sont exposés des lettres et récits de soldats, des insignes et des uniformes identifiés, etc. ${ }^{78}$ Ici encore, ce n'est pas la version de l'état-major que le musée propose aux visiteurs, sans pour autant s'y opposer. La reproduction en cartes postales de photographies du monument, de l'église commémorative, des cérémonies annuelles ou même du musée permet de diffuser à l'échelle nationale des images patriotiques en provenance de la frontière.

Un tourisme mémoriel se développe dans toute la zone frontalière ${ }^{79}$. On estime entre 6000 et 8000 le nombre de participants aux cérémonies du 16 août, avec des pics notables à la suite d'événements politiques majeurs ou lors de la venue de membres du gouvernement $^{80}$. On peut alors compter jusqu'à 25000 personnes (fig. 4). Il faut 
distinguer le pèlerinage, qui est d'ordre religieux, du tourisme qui est d'ordre récréatif ${ }^{81}$, même si la distinction entre les deux pratiques est parfois ténue car le touriste peut se recueillir et le pèlerin vivre un temps de loisir ${ }^{82}$. Cette ambivalence s'observe nettement avec l'ouverture de la crypte aux visiteurs s'acquittant d'un billet de 50 centimes $^{83}$. Alors que se développent les pèlerinages catholiques, les vétérans se rendent souvent en famille sur les lieux des combats ${ }^{84}$. Les récits ne manquent pas des deux côtés de la frontière ${ }^{85}$.

Figure 4

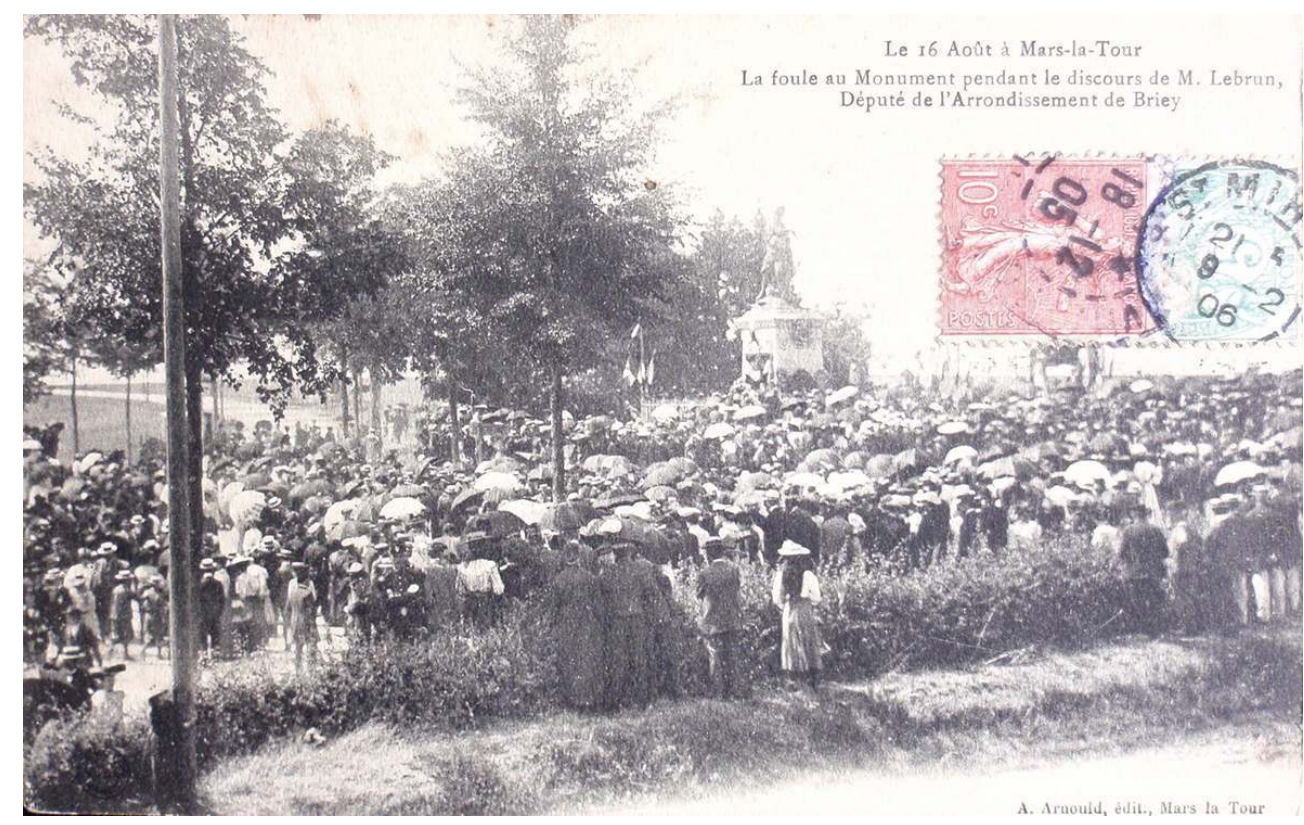

Le 16 août à Mars-la-Tour. La foule au Monument pendant le discours de M. Lebrun, Député de l'arrondissement de Briey, carte postale, A. Arnould édit., Mars-la-Tour, début du Xxe siècle.

De nombreuses cartes françaises des champs de bataille font figurer les tombes des soldats morts en $1870^{86}$, mais c'est sans comparaison avec les très nombreux guides touristiques que produisent les Allemands ${ }^{87}$. Il faut mettre cette profusion en relation avec les spécificités de la commémoration allemande de la guerre, où chaque régiment ou même des individus (des officiers le plus souvent) ont leur propre monument. On en compte un grand nombre rien qu'à Mars-la-Tour, qui possède aussi un cimetière allemand. Les militaires allemands en garnison à Metz sont nombreux à se rendre sur les champs de bataille lors des dates anniversaires des combats, où ils déclament quelques poèmes patriotiques ${ }^{88}$. Parfois même, ils se rendent au monument national français. La règle élémentaire étant pour tout militaire souhaitant se recueillir de l'autre côté de la frontière de ne pas porter l'uniforme ${ }^{89}$; la non-observance de ce principe peut générer de fâcheux incidents ${ }^{90}$.

L'historien doit aussi être attentif aux formes de contestation que peut susciter une telle concentration et célébration du patriotisme, même si elles sont minimes et peu documentées. On déplore par exemple la dégradation des hauts-reliefs du monument de Mars-la-Tour en $1886^{91}$ : les sabres et les épées de plusieurs chefs militaires ont été cassés à coups de pierre ${ }^{92}$. Les raisons de cette détérioration intentionnelle sont inconnues, d'autant qu'elle n'a pas donné lieu à une enquête ${ }^{93}$, mais tout laisse à penser que les auteurs sont des enfants; la dégradation n'est pas un fait isolé dans la région ${ }^{94}$. Les mouvements nationalistes, des deux côtés de la frontière, ne manquent jamais de monter 
en épingle le moindre incident ${ }^{95}$. Ces actes s'inscrivent aussi dans une évolution du sentiment patriotique local qui prend des accents chauvins à la fin du siècle.

\section{«France, entends-tu la dernière prière de tes enfants couchés dans leurs tombeaux ? " ${ }^{96}$, Mars-la-Tour, sentinelle revanchiste?}

À la fin du xix ${ }^{e}$ siècle, le souvenir de la guerre de 1870 s'institutionnalise en France, à travers notamment l'association du Souvenir Français. Les initiatives locales comme celle de Mars-la-Tour, isolées et sans soutien public dans les années 1870, se fédèrent et jouissent désormais de la bienveillance de l'État. Cela est particulièrement visible à la frontière. Les historiens ont voulu voir dans l'inflation commémorative des années 1890 les symptômes d'une fièvre nationaliste revanchiste consécutive à l'épisode boulangiste. Sans remettre en question l'essor nationaliste durant cette période, l'observation minutieuse des pratiques mémorielles en Lorraine permet de qualifier et de mesurer l'attachement à la France des populations frontalières avec plus de nuance.

Pour comprendre l'institutionnalisation de la mémoire des soldats morts lors des combats de 1870-1871, il faut se départir de la vision d'une politique publique mémorielle conduite directement par l'État, pensée dans la capitale et diffusée à l'ensemble du territoire. Le Souvenir Français, association créée en 1887, est crédité de nombreuses réalisations : il recouvre en fait une myriade de comités locaux qui sont les réels protagonistes de l'entretien des tombes des soldats et des projets de monuments. La structure, présidée et fondée par François-Xavier Niessen, se charge de piloter toutes ces initiatives venues d'en bas et d'intercéder en leur faveur auprès de l'État. Le financement reste supporté pour tout ou majeure partie par les souscriptions émises localement ${ }^{97}$. Elle se charge ensuite de pérenniser ces réalisations en les entretenant. Ainsi, le Souvenir Français entretient la crypte et fleurit tous les ans le monument de Mars-la-Tour ${ }^{98}$. L'accroissement des projets commémoratifs n'est pas tant imputable au revanchisme qu'à une plus grande coordination, et bienveillance publique. L'âge d'or de la «statuomanie » - en lien ou non avec le souvenir de la guerre - ne débute qu'avec la républicanisation du régime ${ }^{99}$, lorsque l'État n'use plus d'un pouvoir discrétionnaire en la matière et fait des demandes d'autorisation une simple formalité administrative ${ }^{100}$. Ce qui est nouveau, c'est que les monuments commémoratifs - comme celui de Mars-la-Tour - sortent des cimetières pour devenir dans l'espace public un support de pédagogie patriotique ${ }^{101}$.

La III République est trop libérale pour se doter d'une politique commémorative "en gestion directe "; cependant, l'État conserve son rôle de modération et suit de très près les initiatives qui lui paraissent politiquement trop partisanes. Ainsi, le monument de Baccarat, en 1912, trop marqué par son caractère nationaliste et clérical est-il autorisé, mais n'est pas soutenu par le Souvenir Français ${ }^{102}$. Les autorités politiques et administratives distinguent ce qui relève du patriotisme, attachement «spontané » à la patrie, et du nationalisme, qui mobilise le patriotisme à des fins politiques. Cette conception est si bien partagée par les autorités allemandes qu'elles autorisent, certes après de nombreuses tractations, la construction en Lorraine annexée de plusieurs monuments à l'initiative de comités du Souvenir Français. Lors de l'inauguration du plus important d'entre eux, à Noisseville, à l'est de Metz, en 1908, il est même permis de jouer La Marseillaise !103 Ce soudain libéralisme allemand s'explique par l'intégration croissante 
de l'Alsace-Lorraine à l'Empire, mais nous faisons aussi l'hypothèse que les autorités cherchent à contrer le succès de Mars-la-Tour. En effet, les Messins disposent désormais de «leur» lieu de mémoire de la guerre de 1870, et n'ont plus nécessairement besoin de passer la frontière et de se rendre en France.

Parallèlement, lors des cérémonies de Mars-la-Tour, l'esprit de Revanche est bien présent : des cris hostiles à l'Allemagne, «À bas la Prusse !» ou même « Vengez-nous de 1870 ! ${ }^{104}$ se font entendre aux moments les plus tendus des relations franco-allemandes, mais ce chauvinisme reste isolé. Chaque année, des rumeurs se répandent quant à l'arrestation à la frontière d'agents allemands qui chercheraient à perturber la fête du 16 août, particulièrement lors de grandes crises comme celle de l'affaire Schnæbelé105. L'affluence à Mars-la-Tour et les risques de débordements contraignent à déployer chaque année un important service d'ordre.

Le revanchisme qui peut s'exprimer çà et là n'en reste pas moins sous contrôle, et n'est ni le fruit d'une mobilisation des services de l'État ni de groupes nationalistes, dont aucune trace n'est visible à la frontière ${ }^{106}$. L'incorporation du patriotisme populaire par les élites sociales et politiques permet de réduire la distance entre gouvernants et gouvernés, entre centre et périphérie, et donc précisément de réguler un éventuel nationalisme revanchiste. L'évêque de Nancy enjoint en 1893 à "aimer la France »"107. Le ton est revendicatif et fier, loin du deuil et du dolorisme des années 1870. Le sénateur Mézières déclare, en 1905 : « Pour nous habitants de la frontière, en face de la blessure saignante, les regrets continuent, toujours plus poignants à mesure que les années s'écoulent ${ }^{108}$. »

À la veille de la Première Guerre mondiale, le monument de Mars-la-Tour devient étape du Tour de France. Albert Lebrun, personnalité politique lorraine montante, quant à lui, ne manque jamais la commémoration du 16 août ${ }^{109}$ (voir fig. 4). L'abbé Faller est fait chevalier de la Légion d'honneur en présence de Raymond Poincaré, président du Conseil (et lorrain) ${ }^{110}$. Jamais le dialogue entre l'État et les populations locales, entamé au lendemain de la guerre de 1870 , n'a été aussi fructueux ${ }^{111}$. Poincaré appelle déjà à l'« union sacrée »: «Au pied de ce monument commémoratif viennent expirer les murmures de nos querelles intestines. Tout ici conspire à effacer nos divisions et à nous rapprocher dans une conscience plus nette de notre unité nationale [...].» Et là où on s'attendrait à un peu de prudence diplomatique, plusieurs discours sont adressés aux "frères annexés » dans une grande célébration de l'Armée. À Mars-la-Tour, on se souvient d'une guerre et on en prépare une autre.

Chaque année, à Mars-la-Tour, les Lorrains renouvellent sur l'autel des morts de 1870 l'alliance entre la petite patrie, la grande patrie et la patrie du ciel. Chaque 16 août est une renégociation entre les populations frontalières et les élites sociales et politiques de la nation. Le patriotisme local, méprisé lors de l'inauguration du monument, est progressivement pris en compte par le discours républicain, qui l'oriente vers la poursuite de l'unité nationale et la préparation de la guerre. Si l'on observe un certain revanchisme au tournant du siècle, on ne peut qualifier le patriotisme qui s'exprime d'entièrement revanchiste, et encore moins le réduire à une action de l'État. L'abbé Faller veille à ce que son œuvre patriotique conserve un caractère apolitique et local ${ }^{112}$. Le zèle à « rendre toujours plus éclatante la grande cérémonie funèbre du 16 août qui attire chaque année une grande affluence de pèlerins » lui donne une certaine notoriété, et fait de lui une personnalité lorraine de premier plan, respectée jusqu'à sa mort, quelques mois avant le début de la Première Guerre mondiale ${ }^{113}$. Il ne voit donc pas le bombardement 
délibéré du patrimoine mémoriel de Mars-la-Tour perpétré par les Allemands le 16 août $1914^{114}$.

Une fois la guerre terminée, une mémoire chasse l'autre. La frontière se situe désormais sur le Rhin, bien loin. Pour le $50^{\mathrm{e}}$ anniversaire de la bataille de Mars-la-Tour, en 1920, on déplore qu'il n'y ait plus « les foules immenses d'antan »"115. La cérémonie s'est officialisée, aseptisée, banalisée. Tous les élus lorrains, des conseillers généraux aux sénateurs, sont là ; Albert Lebrun préside. La cérémonie n'est plus qu'une réitération des célébrations passées, avec l'évocation des personnalités présentes et des discours prononcés. La routine mémorielle remplace la commémoration populaire d'avant-guerre; on ne s'écrie plus, on applaudit ${ }^{116}$. La mémoire de la guerre de 1870 en Lorraine s'évapore au profit d'une pluralité de mémoires régionales de la Grande Guerre. Le monument de Mars-laTour perd sa qualité de borne frontalière symbolique face à l'Allemagne et cesse d'être le lieu d'un patrimoine vivant où dialoguent l'État et les populations locales.

\section{NOTES}

1. - TURINAZ, Charles-François. Discours prononcé auprès du monument de Mars-la-Tour à l'occasion du vingtième anniversaire de la bataille de Gravelotte le 16 Août 1890. Nancy : Le Chevallier, 1890, p. 7. 2. - HENRY, Armand, HOUSSEMAND, Bernard et NAUROY, Jean. "Les lieux de mémoire de la guerre de 1870 en pays messin ». Les Cahiers lorrains, ${ }^{\mathrm{n}}$ o3-4, octobre 1992, p. 333-342.

3. - Concernant les combats de la guerre de 1870 en général, et en Lorraine en particulier, voir ROTH, François. La Guerre de 1870. Paris : Fayard, 1990.

4. - KOSELLECK, Reinhart. «Les monuments aux morts, lieux de la fondation de l'identité des survivants ». Dans L'expérience de l'histoire. Paris : Gallimard/Le Seuil, 1997, p. 135-160.

5. - ANDERSOn, Benedict. Imagined communities. Reflections on the origin and spread of nationalism. Londres/New York : Verso, 1983.

6. - TISON, Stéphane. Comment sortir de la guerre ? Deuil, mémoire et traumatisme, 1870-1940. Rennes : PUR, 2011.

7. - PROST, Antoine. "Les monuments aux morts. Culte républicain? Culte civique? Culte patriotique?». Dans NORA, Pierre (dir.). Les Lieux de mémoire. Paris : Gallimard, 1984-1992. T. I, La République, p. 195-225.

8. - Ibid.

9. - Ibid.

10. - DALISSON, Rémi. «Les racines d'une commémoration: les fêtes de la Revanche et les inaugurations de monuments aux morts de 1870 en France (1871-1914)». Revue historique des armées, $\mathrm{n}^{\circ} 274,12$ mai 2014, p. 23-37 ; voir aussi Id. Les Guerres et la mémoire. Enjeux identitaires et célébrations de guerre en France de 1870 à nos jours. Paris : CNRS éd., 2013.

11. - VARLEY, Karine. Under the Shadow of Defeat. The War of 1870-71 in French Memory. Basingstoke : Palgrave Macmillan, 2008.

12. - VAN GINDERACHTER, Maarten et BEYEN, Marnix (dir.). Nationhood from Below. Europe in the Long Nineteenth Century. Basingstoke : Palgrave Macmillan, 2012.

13. - LEROY, Osvald. Mars-la-Tour, 16-18 août 1870. Paris : Fischbacher, 1887, p. 6.

14. - Ibid. 
15. - Ibid.

16. - Ibid.

17. - Ibid., p. 7.

18. - Le curé de Gondreville-Fontenoy se plaint du peu d'enthousiasme de la préfecture vis-à-vis des commémorations (lettre du curé de Gondreville-Fontenoy, 31 juillet 1872, archives départementales de Meurthe-et-Moselle (désormais AD MM) 1 M 670).

19. - LEROY, Osvald. Mars-la-Tour, 16-18 août 1870. Op. cit., p. 7.

20. - Ibid., p. 9.

21. - Ibid., p. 7.

22. - Courrier du maire de Mars-la-Tour, 9 décembre 1875 (AD MM, 1 M 670).

23. - Courrier du secrétaire du comité de souscription du monument de Mars-la-Tour, 25 septembre 1875 ; extrait du registre des délibérations du conseil municipal de Mars-la-Tour du 11 janvier 1896 (AD MM, 1 M 670).

24. - CEuvre créée en 1874 en hommage à l'héroïsme des soldats fraçais durant la guerre de 1870, qui connut un grand succès et une large diffusion.

25. - LA TOUR-DU-PIN-CHAMBLY DE LA CHARCE, René de. L'Armée française à Metz. Paris : F. Amyot, 1871.

26. - FALLER, Joseph. Souscription proposée pour élever une chapelle à Mars-la-Tour en faveur des soldats morts sur de nombreux champs de bataille. Nancy : Imprimerie de Vagner, 1876.

27. - Un optant est un individu ayant choisi de quitter l'Alsace-Lorraine au moment de son annexion par l'Empire allemand, afin de conserver la nationalité française.

28. - Sur l'appropriation visuelle de l'espace par le panorama, voir OETTERMANN, Stephan. Das Panorama. Die Geschichte eines Massenmediums. Francfort-sur-le-Main : Syndikat, 1980.

29. - Photographie de l'église commémorative dans BADEL, Émile. Mars-la-Tour et son monument national. Mars-la-Tour : Ritter-Roscop, 1893.

30. - ANDERSON, Benedict. Imagined Communities. Op. cit.

31. - LEROY, Osvald. Mars-la-Tour, 16-18 août 1870. Op. cit., p. 36.

32. - Ibid., p. 37.

33. - Ibid., p. 9.

34. - Courrier du ministre de l'Intérieur, 21 avril 1875 (AD MM, 1 M 670).

35. - Sur cet épisode méconnu de l'histoire des relations franco-allemandes, voir JANORSCHKE, Johannes. Bismarck, Europa und die « Krieg-in-Sicht »-Krise von 1875. Paderborn : Schöningh, 2010.

36. - Courrier du ministre de l'Intérieur, 14 mai 1875 (AD MM, 1 M 670).

37. - Courrier du préfet de Meurthe-et-Moselle, 15 mai 1875 (AD MM, 1 M 670).

38. - Courrier du préfet de Meurthe-et-Moselle, 31 octobre 1875 (AD MM, 1 M 670).

39. - Courrier du préfet de Meurthe-et-Moselle, $1^{\mathrm{er}}$ novembre 1875 (AD MM, 1 M 670).

40. - Ibid. (AD MM, 1 M 670).

41. - Courrier du préfet de Meurthe-et-Moselle, 15 mai 1875 (AD MM, 1 M 670).

42. - Courrier du préfet de Meurthe-et-Moselle, $1^{\mathrm{er}}$ novembre 1875 (AD MM, 1 M 670).

43. - Courrier du ministre de l'Intérieur, 14 mai 1875 (AD MM, 1 M 670).

44. - Télégramme au préfet de Meurthe-et-Moselle, non daté (AD MM, 1 M 670).

45. - Publication anonyme de l'inauguration du monument élevé à Mars-la-Tour, 1875 (AD MM, $1 \mathrm{M} \mathrm{670).}$

46. - Courrier de l'abbé Faller, 7 septembre 1877 (AD MM, 1 M 670).

47. - Courrier du ministre de l'Intérieur, 11 septembre 1877 (AD MM, 1 M 670).

48. - Courrier du général commandant de la $11^{\mathrm{e}}$ division d'infanterie, 22 septembre 1877 (AD MM, $1 \mathrm{M} \mathrm{670).}$

49. - Courrier du ministre de l'Intérieur, 14 septembre 1877 (AD MM, 1 M 670). 
50. - Courrier du ministre de l'Intérieur, 17 septembre 1877 (AD MM, 1 M 670).

51. - Courrier du chef d'escadron de la compagnie de gendarmerie de Mars-la-Tour, 22 septembre 1877 (AD MM, 1 M 670).

52. - Discours de M. le préfet, marquis de Chambon (LEROY, Osvald. Mars-la-Tour, 16-18 août 1870. Op. cit., p. 18-19).

53. - Discours de M. Noël, chanoine honoraire, curé archiprêtre de Briey (ibid., p. 13-16).

54. - Discours de M. Ladoucette (ibid., p. 19-22) ; Discours de M. Pierson (ibid., p. 22-23).

55. - LEROY, Osvald. Mars-la-Tour, 16-18 août 1870. Op. cit., p. 38.

56. - Ibid.

57. - Courrier du secrétaire du comité de souscription du monument de Mars-la-Tour, 25 septembre 1875 (AD MM, 1 M 670).

58. - Discours de Mgr Foulon, évêque de Nancy (LEROY, Osvald. Mars-la-Tour, 16-18 août 1870. Op. cit ., p. 25-28).

59. - Voir les très belles pages autour de Pro Patria Mori. KANTOROWICZ, Ernst H. « Mourir pour la patrie ». Dans Id. Mourir pour la patrie et autres textes. Éd. Pierre Legendre. Paris : PUF, 1984, p. $105-141$.

60. - Avis relatif à l'anniversaire des 16 et 18 août 1870 adressé à la presse en 1887 (LEROY, Osvald. Mars-la-Tour, 16-18 août 1870. Op. cit., p. 65-66).

61. - Les journaux allemands de Metz critiquent même les autorités d'Alsace-Lorraine, jugées laxistes en la matière.

62. - LEROY, Osvald. Mars-la-Tour, 16-18 août 1870. Op. cit., p. 12-17.

63. - BADEL, Émile. Mars-la-Tour et son monument national. Op. cit., p. 87.

64. - L'Est républicain, 17 août 1907.

65. - VARLEY, Karine. Under the Shadow of Defeat. Op. cit., p. 118.

66. - LEROY, Osvald. Mars-la-Tour, 16-18 août 1870. Op. cit., p. 25.

67. - Courrier de l'abbé Faller et du maire de Mars-la-Tour, 7 septembre 1877 (AD MM, 1 M 670).

68. - L'Est républicain, 18 août 1889.

69. - LEROY, Osvald. Mars-la-Tour, 16-18 août 1870. Op. cit., p. 66.

70. - L'Est républicain, 18 août 1892.

71. - Rémi Dalisson souligne la grande diffusion de ces chansons patriotiques jusqu'à la veille de la Première Guerre mondiale (DALISSON, Rémi. « Les racines d'une commémoration ». Art. cit.).

72. - VARLEY, Karine. Under the Shadow of Defeat. Op. cit., p. 106.

73. - Publication anonyme sur l'inauguration du monument élevé à Mars-la-Tour, 1875 (AD MM, $1 \mathrm{M} \mathrm{670).}$

74. - LEROY, Osvald. Mars-la-Tour, 16-18 août 1870, Op. cit., p. 66.

75. - L'Est républicain, 17 août 1900.

76. - BADEL, Émile. Le Curé de Mars-la-Tour. 16 août 1910. Son œuvre, son musée. S.l., s.n. 1910.

77. - Dossier de légion d'honneur de Joseph Faller (Archives nationales, LH 929/11), consultable dans la base Léonore.

78. - Mars-la-Tour. L'Abbé Faller dans son Musée. Carte postale A. Arnould éd., Mars-la-Tour, début du Xx siècle.

79. - LASSÈRE, Madeleine. « Le $\mathrm{XI}^{\mathrm{xe}}$ siècle et l'invention du tourisme funéraire ». Revue d'histoire moderne et contemporaine, $\mathrm{n}^{\circ} 44-4,1997, \mathrm{p} .601-616$.

80. - LEROY, Osvald. Mars-la-Tour, 16-18 août 1870. Op. cit., p. 17.

81. - COHEN, Erik. «A Phenomenology of Tourist Experiences ». Sociology, vol. 13, no2, 1 Mai 1979, p. 179-201.

82. - WALTER, Tony. «War Graves in Pilgrimage ». Dans READER, Ian et WALTER, Tony (éd.). Pilgrimage in Popular Culture. Basingstoke : Macmillan/Palgrave, 1993, p. 63-91. 
83. - LEROY, Osvald. Mars-la-Tour, 16-18 août 1870. Op. cit., p. 66.

84. - VARLEY, Karine. Under the Shadow of Defeat. Op. cit., p. 112-113.

85. - Un bon exemple de cette circulation touristique : AIRE, Albert d', Mme. Pèlerinage aux champs de bataille de Saint-Privat, Mars-la-Tour et Sainte-Marie-au-Mont. Paris : Téqui, 1893. Voir sur Gallica : https://gallica.bnf.fr/ark:/12148/bpt6k64716630.texteImage [consulté le 06/02/2019].

86. - Cartes des tombes des champs de bataille dans LEROY, Osvald. Mars-la-Tour, 16-18 août 1870. Op. cit., p. 59.

87. - Par exemple BUSSLER, Wilhelm. Metz und die Schlachtfelder. Praktischer Führer. Metz : Muller, 1897.

88. - DITTRICH, Max. Deutsche Heldengraeber im Reichslande. Rathenow : Max Babenzien, 1895.

89. - Rapports de police, 3 juillet 1896 (AD MM, 4 M 219).

90. - Dossier sur une violation de frontière près d'Habonville le 6 juin 1892 (AD MM, 4 M 176).

91. - LEROY, Osvald. Mars-la-Tour, 16-18 août 1870. Op. cit., p. 37.

92. - Ibid.

93. - Karine Varley l'attribue à la grande proportion de femmes et d'enfants qui en sont à l'origine (VARLEY, Karine. Under the Shadow of Defeat. Op. cit., p. 62-63).

94. - Un jeune Lorrain de 15 ans retourne des croix en bois marquant des tombes de soldats allemands en 1896. Rapport de police, 4 décembre 1896 (AD MM, 4 M 194).

95. - Lire la description horrifiée d'Henri Galli, membre de la Ligue des patriotes, en 1894, d'un pique-nique allemand sur des tombes militaires (VARLEY, Karine. Under the Shadow of Defeat. Op. cit., p. 120-121).

96. - Extrait de VILLEMER, Gaston, NAZET, Henri et TAYOUX, Ben. Alsace et Lorraine, 1871. Chant patriotique appartenant au répertoire joué lors des cérémonies du 16 août.

97. - Discours du président du comité du Souvenir Français de Longwy lors de l'inauguration du monument aux défenseurs de la place de Longwy, 25 août 1912 (AD MM, 1 M 670).

98. - BADEL, Émile. Mars-la-Tour et son monument national. Op. cit., p. 58.

99. - AGULHON, Maurice. "La "statuomanie" et l'histoire ». Dans Id. Histoire vagabonde [1988]. Paris : Gallimard, 1996, p. 138-185.

100. - FLEURY, Daniel. «Plaques, stèles et monuments commémoratifs : l'État et la "mémoire de pierre" ». Revue historique des armées, ${ }^{\mathrm{n}} \mathrm{o} 259,15$ juin 2010, p. 56-66.

101. - VARLEY, Karine. Under the shadow of defeat, Op. cit., p. 105.

102. - Rapports de police, 25 mai 1912 et 26 juin 1912 (AD MM, 1 M 670).

103. - La politique suivie est exactement la même en Basse-Alsace avec l'inauguration du monument de Wissembourg. Rapports de police, 19 octobre 1909 (AD MM, 1 M 670).

104. - L'Est républicain, 18 août 1892.

105. - L'Est républicain, 18 août 1889. L'affaire Schnæbelé est un important incident diplomatique entre la France et l'Allemagne survenu après l'arrestation du commissaire de police Guillaume Schnæbelé, par des policiers allemands ayant violé le territoire français.

106. - Karine Varley montre à quel point le rôle de groupes nationalistes comme la Ligue des patriotes dans les commémorations de la guerre a été surévalué en raison d'une presse nationaliste réactive et de l'effet de loupe de Paris (VARLEY, Karine. Under the Shadow of Defeat. Op. cit., p. 144-151).

107. - Le discours est titré «Il faut aimer la France» (TURINA z, Charles-François. Discours patriotiques. Nancy : E. Drioton, 1900, p. 197-208).

108. - L'Est républicain, 17 août 1905.

109. - L'Est républicain, 17 août 1907.

110. - L'Illustration, 19 août 1910.

111. - Voir BARRAL, Pierre. L'Esprit lorrain. Cet accent singulier du patriotisme français. Nancy: Presses universitaires de Nancy, 1989. 
112. - Dossier de légion d'honneur de Joseph Faller (Archives nationales, LH 929/11). Voir cidessus note 77.

113. - Ses funérailles furent prétexte à une grande cérémonie patriotique comparable aux commémorations du 16 août.

114. - Journal de Meurthe-et-Moselle, 20 août 1914.

115. - L'Est républicain, 17 août 1920.

116. - L'Est républicain, 17 août 1920.

\section{RÉSUMÉS}

Le monument de la guerre franco-allemande de 1870, situé à Mars-la-Tour en Meurthe-etMoselle, commémore les soldats français tombés lors des combats des 16 et 18 août 1870. Sculpté par Frédéric-Louis-Désiré Bogino, édifié par souscription nationale à l'initiative des habitants, il est inauguré en 1875. Le monument, auquel se joignent une église transformée en temple patriotique et un musée, constituent un patrimoine qui devient très vite le support d'un culte des morts et d'un tourisme mémoriel. Tous les ans, jusqu'en 1914, des cérémonies entretiennent le souvenir des disparus et attirent des milliers de participants. Véritable centre de la mémoire locale, le monument de Mars-la-Tour a la particularité de se situer à moins d'un kilomètre de la frontière qui sépare la France de l'Allemagne entre 1871 et 1918. Le présent article a plusieurs objectifs: contribuer à l'histoire générale de la patrimonialisation des frontières, revisiter l'historiographie des commémorations de guerre en France, enfin, rendre compte de la genèse d'un monument singulier en Lorraine en interrogeant les pratiques qui l'ont accompagné. Il s'agit ici de se dépayser d'une approche par le haut et de porter l'attention sur les acteurs locaux à la frontière.

Located in the village of Mars-la-Tour in the department of Meurthe-et-Moselle, an 1870 war memorial, inaugurated in 1875, commemorates the French soldiers who fell at nearby battlefields during the fighting between 16 and 18 August 1870. It was sculpted by Bogino and erected after a national fundraising campaign launched by local inhabitants. This monument, with the later addition of a church and a local museum dedicated to the 1870 war, soon became an important landmark for the cult of the dead. It also became an attraction for memory-based tourism. Ceremonies took place there every year until 1914, attracting thousands of participants who commemorated the memory of the dead. The Mars-la-Tour monument became an authentic centre for local remembrance. One of its specificities was its location, only one kilometre from the new border separating France from Germany between 1871 and 1918. This paper has several aims: to offer a contribution to the history of border memorials; to revisit the historiography of French war commemorations; and, finally, to study the genesis of a specific monument in Lorraine, focusing on social practices as seen from below. The ambition is to avoid the top-down approach of heritage recognition processes and to pay attention to the local logics and actors at the border. 
INDEX

Mots-clés : patrimoine, frontière, monument, monuments aux morts, mémoire, souvenir, commémoration, musée, Lorraine, nation, nationalisme, guerre de 1870, patrie, patriotisme, Mars-la-Tour

Keywords : heritage, frontier, monument, war memorials, memory, souvenir, commemoration, museum, Lorraine, nation, nationalism, Franc-Prussian war of 1870-1871, country, fatherland, patriotism, Mars-la-Tour

\section{AUTEUR}

\section{BENOÎT VAILLOT}

Doctorant en histoire EHESS / Institut Universitaire Européen / Unistra (Arche EA 3400) https:// me.eui.eu/benoit-vaillot/ benoit.vaillot@eui.eu 